\title{
Frequency of Serological Markers of Chlamydia trachomatis in Cases of Ectopic Pregnancy in Antananarivo Madagascar
}

\author{
Randriamahazo Rakotomalala Toky ${ }^{1, *}$, Anjaharisoniaina Narindra Tatiana ${ }^{2}$, \\ Razafindraibe Andriatompoina Felanarivo ${ }^{2}$, Andrianampanalinarivo Rakotovao Hery ${ }^{2}$, \\ Rasamindrakotroka Andry ${ }^{1}$ \\ ${ }^{1}$ Laboratory of medical biology, Befelatanana Obstetric Gynecological University Hospital (BOGUH), Antananarivo, Madagascar \\ ${ }^{2}$ Gynecology service, Befelatanana Obstetric Gynecological University Hospital (BOGUH), Antananarivo, Madagascar
}

\section{Email address:}

drrtoky@yahoo.fr (R. R. Toky), anjatatiana@yahoo.fr (A. N. Tatiana), rivorazafin@gmail.com (R. A. Felanarivo), heryrakotovao.univ.tana@gmail.com (A. R. Hery), andryrasamindrakotroka@hotmail.com (R. Andry)

${ }^{*}$ Corresponding author

\section{To cite this article:}

Randriamahazo Rakotomalala Toky, Anjaharisoniaina Narindra Tatiana, Razafindraibe Andriatompoina Felanarivo, Andrianampanalinarivo Rakotovao Hery, Rasamindrakotroka Andry. Frequency of Serological Markers of Chlamydia trachomatis in Cases of Ectopic Pregnancy in Antananarivo Madagascar. American Journal of Laboratory Medicine. Vol. 2, No. 5, 2017, pp. 99-103. doi: 10.11648/j.ajlm.20170205.14

Received: July 5, 2017; Accepted: August 4, 2017; Published: October 24, 2017

\begin{abstract}
Introduction: Chlamydia trachomatis is a bacteria responsible of the most prevalent sexually transmitted disease worldwide, causing severe tubal damage responsible of ectopic pregnancy. This study was conducted to determine the incidence of chlamydia in women with ectopic pregnancy ruptured in a reference hospital of capital town of Madagascar. Patients and methods: This is a transversal and descriptive study of screening for Chlamydia trachomatis IgG cases among ectopic pregnancy seen at the University Hospital of Obstetrics Gynecology Befelatanana (CHUGOB) from 01 February to 30 May 2015. Results: During the study period, we recorded 41 cases with a overall rate of ectopic pregnancy of $2.04 \%$ when reported to the total number of deliveries. (n: 2347) The average age of patients was 30.50 years with predominance in primiparous (66\%). More than half of the patients had at least one risk factor and $90 \%$ had a history of abortion induced and spontaneous miscarriage. The frequency of positive Chlamydia trachomatis IgG is $20.83 \%$ (n: 10) among which 7 presented pathological fallopian tube. Conclusion: The best way to avoid complications is precocious screening, but the cost of the screening tests remains an obstacle in developing countries like Madagascar.
\end{abstract}

Keywords: Chlamydia trachomatis, Ectopic Pregnancy, Madagascar

\section{Introduction}

Chlamydia trachomatis is a bacterium responsible for sexually transmitted infections (STIs) with almost 100 million new cases per year worldwide [1]. Chlamydia trachomatis infection is often asymptomatic favoring the delay of diagnosis, the transition to chronicity and so on to complications. Moreover, it is a public health issue due to the severity of sequestration: in tubal lesions which cause irreversible sequelae (tubal hypofertility, or chronic pelvic pain) and responsible for ectopic pregnancy (EP). The ectopic pregnancy is defined as the implantation and development of the fertilized egg outside the uterine cavity and represents the first cause of death in the first trimester of pregnancy. Moreover, EP significantly compromises later fertility [2]. Interestingly, EP rate of women with Chlamydia trachomatis infections is around 10\%, compared with $1.4 \%$ to $2 \%$ for women in the general population [3]. In Madagascar, no relevant studies on the prevalence of Chlamydia trachomatis infection in women have been reported so far. The present study aims to evaluate the incidence of Chlamydia trachomatis infection among women with ectopic pregnancy and subsequently evaluate of the correlations between Chlamydia serology and the macroscopic aspect of the fallopian tubes. 


\section{Materials and Methods}

This is a transversal descriptive and analytical study of cases of ectopic pregnancy seen at the Befelatanana Obstetric Gynecological University Hospital (BOGUH). Our study covers a period of four months (01 February to 30 May 2015). It is represented by all women of childbearing age admitted to BOGUH. We included all women of childbearing age who had a ruptured, diagnosed and treated uterine pregnancy at BOGUH. We excluded from the study patients treated or operated on suspicion of ectopic pregnancy and whose diagnosis was erroneous during surgery and ectopic pregnancies treated medically. We used a simple exhaustive type sampling method and the counting was made from a pre-established fact sheet comprising several study parameters: such as age, parity, provenance, mode of admission, occupation, marital status, number of partners, occupation, history but also clinical parameters such as functional, physical and general signs. The data were gathered from the interviewing of patients and the consultation of their medical records after their oral consent and the agreement signed by the patient. All women included in the study were screened for Chlamydia trachomatis Ig G (Immunoglobulin G) Enzyme Immunoassay MEDIFF® France. According to the manufacturer's recommendations, Chlamydia trachomatis Ig G index: $\geq 1$ and negative if Chlamydia trachomatis Ig G index: $\leq 0.99$. The analysis of the data was carried out with the software R. Measures were taken for strict confidentiality during the preparation of the files.

\section{Results}

During the period 1 February to 31 May 2015, we collected 48 cases of EP out of 2347 deliveries achieving a frequency of $2.04 \%$. EP accounted for $6.20 \%$ of indications for surgical procedures (gynecological and obstetric) at BOGUH during this period, 48 cases among 773 procedures. It accounted for $20.08 \%$ of gynecological procedures. We excluded two cases of medically treated EP and three patients suspected of having suspected EP but whose diagnosis was incorrect during surgery (two pyosalpinx and one ovarian cyst rupture). The average age of our patients was 30.50 years. The extreme ages were 19 years and 40 years. The mean gravidity was 3.27 . We found an average parity of 1.7 . Primipares were the most affected with a rate of $33.33 \%$ (16 cases) followed by paucipares which were $31.25 \%$ of the cases. Married women and couples in concubinage accounted for $47.92 \%$ of the cases, while singles constituted only $4.17 \%$. Only $12.5 \%$ of our patients had a university degree and $35.52 \%$ had a housekeeping profession. We had 32 patients, or $66.66 \%$, with at least one risk factor for EP compared with 16 patients is $33.33 \%$ without a risk factor. Induced abortion and spontaneous miscarriage accounted for $97.90 \%$ of cases. Fifty percent had more than two sexual partners.

In the clinical aspect of the patients during admission, it is dominated to $97.92 \%$ by induced pelvic pain, with positive Douglas puncture in $72.91 \%$ of cases. Pelvic ultrasound finds in $72.92 \%$ of cases in empty uterus and a latero-uterine mass. The serology for anti-IgG antibodies Chlamydia trachomatis is positive in $20.83 \%$ ( $\mathrm{n}=10$ patients). During surgery, ampullar localization predominated with $70.83 \%$ of cases and the aspect of the contralateral fallopian tube is healthy in $50 \%$ of cases, but the adhesion is the predominant lesion in $20.83 \%$. There is no significant statistical correlation between the appearance of the contralateral fallopian tube and the results of the serology $(p=0.155)$ (Table 1) but also the aspect of the contralateral fallopian tube and the number of partners $(\mathrm{p}=0.88)$ (Table 2$)$.

Table 1. Distribution of patients according to the aspect of the contralateral fallopian tube and serology IgG Chlamydia trachomatis.

\begin{tabular}{llllll}
\hline Serology & \multicolumn{4}{l}{ Healthy fallopian tube } & \multicolumn{3}{l}{ Pathological fallopian tube } & P value \\
\hline & Effective & Rate (\%) & Effective & Rate (\%) & \\
\hline Negative & 21 & 87,50 & 17 & 70,83 & 0,155 \\
Positive & 3 & 12,50 & 7 & 29,17 & \\
\hline
\end{tabular}

Table 2. Distribution of patients according to the aspect of the contralateral fallopian tube and the number of partners.

\begin{tabular}{llllll}
\hline Number of partners & \multicolumn{2}{l}{ Healthy fallopian tube } & \multicolumn{2}{l}{ Pathological fallopian tube } & P value \\
\hline & Effective & Rate (\%) & Effective & Rate (\%) & \\
\hline 1 & 12 & 50 & 12 & 50 & 0.881 \\
$\geq 2$ & 9 & 37,50 & 10 & 41,67 & \\
$\geq 4$ & 3 & 12,50 & 2 & 8,33 & \\
\hline
\end{tabular}

\section{Discussion}

In Madagascar, few studies have been reported on the frequency of Chlamydia trachomatis infection, the majority of studies have been carried out among sex workers with a rate of $12 \%$ to $17 \%$ in the province of Antananarivo [4 ] [5]. The present study is the first in estimating the prevalence of Chlamydia trachomatis in women with ruptured ectopic pregnancy. The frequency of EP in our hospital remains more or less stable at about $2 \%$ [6]. This frequency is similar to that of some African countries like Ville, Gabon (2.30\%) but low compared to those found in Ouagadougou, Burkina Faso $(3.50 \%)$ and those of the Monastir University Hospital in Tunisia $(5.10 \%)$. Genital infection Chlamydia trachomatis is a common condition, affecting mainly young subjects, between 15 and 25 years old. It is mostly asymptomatic and can be screened in populations at risk [1]. Chlamydia trachomatis is a common pathogen of pelvic inflammatory 
disease (PID), and is isolated in the upper genital tract of about one-quarter of the PID patients. Among untreated Chlamydia trachomatis positive women, studies have reported that $2.0 \%$ to $4.5 \%$ developed clinical PID in about 14 days [7]. In a prospective study of 1170 women from five American sites, $19 \%$ of people with Chlamydia trachomatis diagnosed with PCR developed clinical PID within three years [8]. The World Health Organization estimates that 92 million new cases of Chlamydia trachomatis occur every year in the world. An estimated 3-4 million new cases occur each year in the United States, 5 million in Western Europe, and 16 million in sub-Saharan Africa [9] [10] [11]. The prevalence of Chlamydia trachomatis infection in women is $15 \%$ to $20 \%$ in high-risk populations and $3 \%$ to $7 \%$ in the general population and $9.5 \%$ in students [12] [13].

In a study carried out by Oakeshott et al. found an overall infection rate of Chlamydia trachomatis of $2.5 \%$ in more than 1,000 pregnant women tested and $7.9 \%$ in the population aged 18-24 years [14]. The incidence of upper genital infections was $1.3 \%$ in the detected-treated group and $1.9 \%$ in the control group. Among women with a positive Chlamydia trachomatis test, $9.7 \%$ of the women in the control group had IGH compared with only $1.6 \%$ in the treated group $(p=0.07)$, which is in favor of screening. Immunofluorescence studies in Brazil show a higher prevalence of $56.4 \%$ IgG Chlamydia trachomatis antibodies in women with hypofertility following a history of chronic salpingitis, tubal sterility, or uterine pregnancy [15] [16]. These same rates were found by the same technique in a study carried out in Franceville (Gabon). IgG antibodies were detected in $84 \%$ of women in the symptomatic group, and $53 \%$ and $39 \%$, respectively, of women in two control groups $(\mathrm{P}<0.0001)$. Similarly, IgA antibodies were detected in $62 \%$ of women in the symptomatic group and $31 \%$ and $29 \%$, respectively, of women in two control groups $(\mathrm{P}<0.01)$ [17]. Another study also found higher prevalence of Chlamydia trachomatis antibodies of $84 \%$ in women with ectopic pregnancy with macroscopic tubal abnormalities, compared with women giving birth to full-term infants [18]. In our study, antibodies against Ig G Chlamydia trachomatis were $20.83 \%$ in women with ectopic pregnancy. However, several studies have shown that there is no good evidence for the risk of ectopic pregnancy after infection with Chlamydia trachomatis [3].

Comparing the pregnancy rate in adolescents with and without Chlamydia infection, Wallace et al. reported no significant difference in subsequent pregnancies [19]. In vietnam a case control study that investigated the seroprevalence and molecular evidence of Chlamydia trachomatis in ectopic pregnancies (EP) and uneventful control pregnancies. Where as presence of $C$. trachomatis IgG was strongly associated with EP [adjusted odds ratio (OR) 5·41, 95\% confidence interval (CI) 2.58-11·32], its DNA remained undetected in all tubal lesions [20]. Actually, risk factors for STIs with Chlamydia trachomatis are well identified such as age less than 25 years, occasional sexual partner, more than 2 partners in the year, same-sex partners, absence of diploma, sexual intercourse with one age and history of STI [1]. In our study, more than half of the patients had at least one risk factor.

The immune response to Chlamydia trachomatis infection plays an important role in the pathogenesis of infection. Deep infections such as salpingitis, infertility, ectopic pregnancy, venereal lymphogranuloma, and arthritis are accompanied by an increase in antibodies that can be used in diagnosis. There is a correlation between antibody titers and the severity of tubal inflammation, but a high level of antibodies may persist for several years even after antibiotic therapy [21] [22] [23]. Serology is more involved in the spread of infection than in the diagnosis of infection [24]. It is unnecessary to follow the kinetics of the IgG because, given the evolution of the disease and the numerous recontaminations, it is impossible to demonstrate a seroconversion or a significant increase of antibodies.

If the presence of antibodies is not synonymous with complicated infection, their absence excludes it. A high level of IgG is significant from past or ongoing infection. Given the persistence of antibodies, it is impossible to distinguish a past infection cured from persistent infection one. After treatment, the antibodies persist at a high level for several months and the serology does not allow monitoring the evolution of the disease, so it is not used to check antibody titer after treatment. Its management is limited to the following indications in men and women: suspicion of PID, suspicion of venereal lymphogranulomatosis (genital ulceration, proctitis), a balance of hypofertility of the couple and diagnosis of reactive arthritis or Fiessinger-Leroy-Reiter syndrom. The risk of developing tubal infertility after a PID with Chlamydia trachomatis is between 10 and 20\%. [25] This rate is doubled or even tripled in those who had more than three infections with Chlamydia trachomatis [26]. It's clear that factors related to the virulence of the infecting strain and host factors have an impact on the severity of the disease [27]. There are currently no biological markers predictive of severity and the main obstacle is the absence of technique enable to detect the antecedents in the fallopian tube. The search for Chamydia trachomatis-specific antiChsp60 antibodies has been proposed as a marker of persistent infection [28]. Studies have shown a significant correlation between the presence of anti-Chsp60 antibodies and pelvic infection or tubal obstruction [29]. Elisa tests using these Chsp60 as antigens have been developed with poor specificity [30]. Markers for differentiating a low infection from a high infection were identified by western blot with a sensitivity of $80 \%$ and a specificity of $89 \%$. This work presents an interesting basis for the development of a serodiagnostic test specific to a high infection [31]. Given the severity of the complications of Chlamydia trachomatis infection, screening recommendations in asymptomatic individuals is adopted in several countries [32]. This study is certainly a first in Madagascar; it is limited by the lack of financial means that do not allow the use of molecular techniques in the diagnosis and our inability to conduct a case control study like other countries. 


\section{Conclusion}

The incidence of Chlamydia trachomatis infection among women with ectopic pregnancy in BOGUH remains quite low compared to data from other countries. We did not find a significant statistical correlation between the appearance of the contralateral fallopian tube and the results of the serology.

We suggest an extensive study in the general population associated with serological and molecular techniques will confirm these traits. These studies will help target populations at risk to facilitate screening which is the best way to avoid complications.

\section{References}

[1] Price, M. J., Ades, A. E., Soldan, K., Welton, N. J., Macleod, J., Simms, I., \& Horner, P. J. (2016). The natural history of Chlamydia trachomatis infection in women: a multi-parameter evidence synthesis.

[2] Butts S, Sammel M, Hummel A, Chittams J, \& Barnhart K. Risk factors and clinical features of recurrent ectopic pregnancy: a case control study. Fertility and sterility 2003; 80(6), 1340-1344.

[3] Shaw JLV, Dey SK, Critchley HOD, \& Horne AW. Current knowledge of the aetiology of human tubal ectopic pregnancy. Human reproduction update 2010; dmp057.

[4] Harijaona V, Ramambason JD, Morisset R, Rasamindrakotroka A \& Ravaoarinoro M. Prevalence of and risk factors for sexually-transmitted infections in hidden female sex workers. Médecine et Maladies Infectieuses 2009; 39, 909-913.

[5] Behets FM, Rasolofomanana JR, Van Damme K et al. Evidence-based treatment guidelines for sexually transmitted infections developed with and for female sex workers. Tropical Medicine \& International Health 2003, 8(3), 251258.

[6] Ratinahirana S, Razanamparany PV, Radaniarison $\mathrm{H}$, Ratsimanohatra E, \& Rakotozafy G. Aspects actuels de la grossesse extra-utérine à Nosy Be (Madagascar), de novembre 1993 à février 1995. Cahiers d'études et de recherches francophones/Santé 1997, 7(1), 19-23.

[7] Ness R, Randall H, Richter H, et al. Condom use and the risk of recurrent pelvic inflammatory disease, chronic pelvic pain, or infertility following an episode of pelvic inflammatory disease. Am J Public Health. 2004; 94(8):1327-1329.

[8] Bachmann L, Richey C, Waites K, Schwebke J, Hook III WE. Patterns of Chlamydia trachomatis testing and follow-up at a University Hospital Medical Center. Sex Transm Dis. 1999; 26 (9):496-509.

[9] Ness R, Smith K, Chang C, Schisterman E, Bass D. Prediction of pelvic inflammatory disease among young, single, sexually active women. Sex Transm Dis. 2006; 33 (3):137-142.

[10] Taylor B D, Haggerty, C L. Management of Chlamydia trachomatis genital tract infection: screening and treatment challenges. Infect Drug Resist 2011, 4(1), 19-29.

[11] World Health Organization Global prevalence and incidence of selected curable sexually transmitted infections: Overview and estimates. Available from:

http://whqlibdoc.who.int/hq/2001/WHO HIV AIDS 2001.02 .pdf. Accessed Jul 15, 2010.

[12] Imai H, Nakao H, Shinohara H, et al. Population-based study of asymptomatic infection with Chlamydia trachomatis among female and male students. Int J STD AIDS. 2010; 21(5): 362-366.

[13] Miller W, Ford C, Morris M, et al. Prevalence of chlamydial and gonococcal infections among young adults in the United States. JAMA. 2004; 291(18): 2229-2236.

[14] Oakeshott P, Kerry S, Aghaizu A et al. Randomised controlled trial of screening for Chlamydia trachomatis to prevent pelvic inflammatory disease: the POPI (prevention of pelvic infection) trial. BMJ 2010; 340: c1642.

[15] Machado ACS, Guimarães EMB, Sakurai E, Fioravante FCR., Amaral W N, Alves, MFC. High titers of Chlamydia trachomatis antibodies in Brazilian women with tubal occlusion or previous ectopic pregnancy. Infectious diseases in obstetrics and gynecology, 2007.

[16] Kihlstrom, R. Lindgren, and G. Ryden, Antibodies to Chlamydia trachomatis in women with infertility, pelvic inflammatory disease and ectopic pregnancy, European Journal of Obstetrics \& Gynecology and Reproductive Biology 1990; vol. 35, no. 2-3, pp. 199-204,

[17] Leclerc A, Frost E, Collet M, Goeman J, Bedjabaga L. Urogenital Chlamydia trachomatis in Gabon: an unrecognised epidemic. Genitourinary medicine 1998, 64(5), 308-311.

[18] Ville Y, Leruez M, Glowaczower E, Robertson JN, Ward ME. The role of Chlamydia trachomatis and Neisseria gonorrhoeae in the aetiology of ectopic pregnancy in Gabon. BJOG: An International Journal of Obstetrics \& Gynaecology 1991, 98(12), 1260-1266.

[19] Wallace, L. A., Scoular, A., Hart, G., Reid, M., Wilson, P., \& Goldberg, D. J. (2008). What is the excess risk of infertility in women after genital chlamydia infection? A systematic review of the evidence. Sexually transmitted infections, 84(3), 171175 .

[20] Hornung, S., Thuong, B. C., Gyger, J., Kebbi-Beghdadi, C., Vasilevsky, S., Greub, G., \& Baud, D. (2015). Role of Chlamydia trachomatis and emerging Chlamydia-related bacteria in ectopic pregnancy in Vietnam. Epidemiology \& Infection, 143(12), 2635-2638.

[21] Land JA, Van Bergen JEAM, Morre SA, Postma, MJ. Epidemiology of Chlamydia trachomatis infection in women and the cost-effectiveness of screening. Human reproduction update 2010, 16(2), 189-204.

[22] Akande VA, Hunt LP, Cahill DJ, Caul EO, Ford WCL, Jenkins JM. Tubal damage in infertile women: prediction using chlamydia serology. Hum Reprod 2003; 18:1841-1847.

[23] Gijsen AP, Land JA, Goossens VJ, Slobbe MEP, Bruggeman CA. Chlamydia antibody testing in screening for tubal factor subfertility: the significance of $\operatorname{IgG}$ antibody decline over time. Hum Reprod 2002; 17:699-703.

[24] Stephens AJ, Aubuchon M, Schust DJ. Antichlamydial antibodies, human fertility and pregnancy wastage. Infect Dis Obstet Gynecol 2011. 
[25] Van Valkengoed IGM, Morre' SM, van den Brule AJC, Meijer CJLM, Bouter LM, Boeke AJP. Overestimation of complication rates in evaluations of Chlamydia trachomatis screening programmes implications for cost-effectiveness analyses. Int J Epidemiol 2004; 33:416-425.

[26] Evans C, Das C, Kinghorn G. A retrospective study of recurrent chlamydia infection in men and women: Is there a role for targeted screening for those at risk? Int J STD AIDS. 2009; 20(3):188-192.

[27] Abdelsamed H, Peters J, Byrne GI. Genetic variation in Chlamydia trachomatis and their hosts: impact on disease severity and tissue tropism. Future Microbiol 2013; 8:1129—46.

[28] Rank RG, Dascher C, Bowlin AK, Bavoil PM. Systemic immunization with Hsp60 alters the development of chlamydial ocular disease. Invest Ophthalmol Vis Sci 1995; $36: 1344-1351$.

[29] Peeling RW, Kimani J, Plummer F, Maclean I, Cheang M, Bwayo J, et al. Antibody to chlamydial hsp60 predicts an increased risk for chlamydial pelvic inflammatory disease. J Infect Dis 1997; 175:1153—8.

[30] Eckert LO, Hawes SE, Wölner-Hanssen P, Money DM, Peeling RW, Brunham RC et al. Prevalence and correlates of antibody to chlamydial heat shock protein in women attending sexually transmitted disease clinics and women with confirmed pelvic inflammatory disease. Journal of Infectious Diseases 1997, 175(6), 1453-1458.

[31] De Barbeyrac B, Obeniche F, Peuchant O, Bébéar C. Méthodes de diagnostic des infections à Chlamydiae: directes et/ou sérodiagnostic? Que choisir?. Journal des Anti-infectieux 2014, 16(4), 185-191.

[32] Bakken I, Ghaderi S. Incidence of pelvic inflammatory disease in a large cohort of women tested for Chlamydia trachomatis: A historical follow-up study. BMC Infect Dis. 2009; 9: 130 . 